\title{
Die proximale Femurfraktur
}

\author{
Häufigkeit, Demographie, Ätiologie, Prophylaxe
}

Rolf Balk, Friedrich Hahn, Bogdan Tarcea

\section{Zusammenfassung}

Hüftnahe Femurfrakturen sind nach distaler Radius- und Sprunggelenkfraktur die dritthäufigste Frakturlokalisation beim Erwachsenen, mit einer jährlichen Inzidenz von ca. 573/ 100000 Personen in der BRD und weiter steigender Tendenz durch die demographische Entwicklung mit stetig zunehmender Zahl alter Menschen in der Gesellschaft. Aufgrund der Häufigkeit und hohen Komplikationsmöglichkeiten bereits in der Akutversorgung, stellen die proximalen Femurfrakturen eine große Herausforderung für die Unfallchirurgie dar. Als typische Altersfraktur jenseits der 5.Lebensdekade sind meist multimorbide Patienten betroffen, die Fraktur wird zum ersten Hinweis auf die Dekom- pensation des bisherigen Gesundheits- und Lebenszustandes. Trotz adäquater chirurgischer Versorgung erreichen nur ca. 50\% der Patienten den Ausgangszustand wieder, über ein Drittel verstirbt innerhalb des ersten Jahres. Es gilt wesentliche Frakturrisiken (Osteoporose, Sturzneigung alter Menschen, u.a.) frühzeitig zu erkennen und entsprechend prophylaktisch anzugehen. Der Unfallchirurg, bisher eher mit operationstaktischen Überlegungen beschäftigt, muss sich in Zukunft vermehrt präventiv auch mit Risikoerkennung und Frakturprophylaxe auseinandersetzen, will er nicht im zunehmenden Spannungsfeld zwischen begrenzten finanziellpersonellen Ressourcen und steigendem Patientenaufkommen zerrieben werden.

\section{Einleitung}

Die operative Versorgung von Frakturen am proximalen Femur ist alltägliche Aufgabe jeder unfallchirurgischen Klinik. Bis zu $10 \%$ aller unfallchirurgischen Operationen betreffen diese Diagnose. Trotz aller Routine zählen die knöchernen Verletzungen am proximalen Femur nach wie vor zu den Problemfrakturen mit postoperativen Komplikationsraten bis über $20 \%$ (15). Daran haben auch langjährige intensive Forschung mit Fortschritten in der Implantatentwicklung, der operativen Technik sowie der differenzierten Indikationsstellung bescheiden wenig zu ändern vermocht. Exemplarisch sei hier an die unkalkulierbare Femurkopfnekrose nach Osteosynthese medialer Schen-

OP-JOURNAL 2002; 17: 80-84

(c) Georg Thieme Verlag Stuttgart · New York kelhalsfrakturen oder an die leider nicht seltenen Implantatbrüche und Pseudarthrosen nach instabilen Brüchen im Trochanterbereich erinnert.

\section{Einteilung/Klassifikation}

Eine erste Unterteilung der Frakturen am proximalen Femur in extra- und intrakapsuläre Typen wurde bereits 1884 durch Halstedt veröffentlicht (vor Entdeckung der Röntgenstrahlen 1895!). Eine ähnliche Grobgliederung in Schenkelhalsfrakturen und Frakturen am metaphysären Femur (per- bis subtrochanter) ist auch heute noch im klinischen Alltag gebräuchlich (Abb.1).

$\mathrm{Zu}$ definitiver Therapieplanung und Prognoseabschätzung auch frakturtypischer Komplikationen sind aber differenziertere Klassifikationen (AO-Klassifikation, Pauwels- oder Garden-Einteilung der Schenkelhalsfrakturen) notwendig.

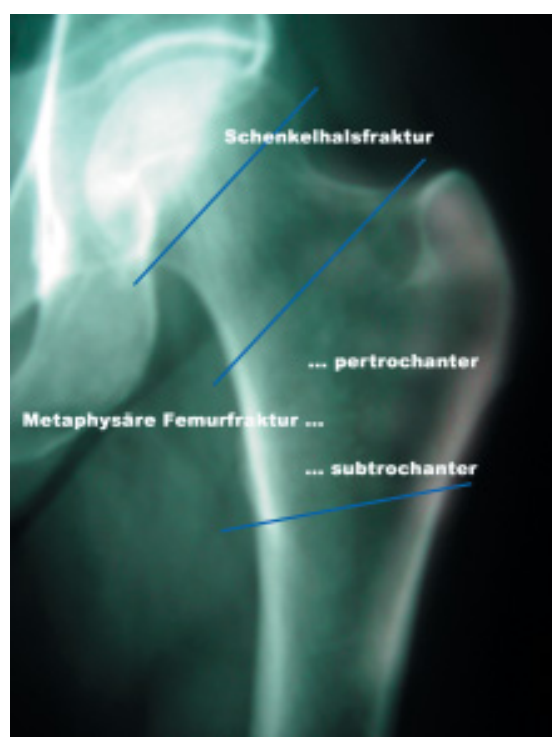

Abb.1 Klinische Unterteilung der proximalen Femurfrakturen.

\section{Häufigkeit, Demografische Entwicklung}

\section{Aktueller Stand}

Bei Auswertung der aktuellen Literatur findet sich eine sehr unterschiedliche Datenlage zur Häufigkeit.

Für die Bundesrepublik Deutschland gab Pfeiffer et al. [7] in einer großen repräsentativen Stichprobe aus 1648000 Versicherten von Betriebskrankenkassen (entspricht ca. 2\% der deutschen Bevölkerung) eine jährliche Inzidenz von 573 Frakturen pro 100000 Einwohner für das Jahr 1996 an.

Bezogen auf die Lokalisation verteilen sich die Frakturen etwa nachfolgend [1]:

- Schenkelhalsfraktur medial 45\%

- Schenkelhalsfraktur lateral $12 \%$

- Femurfraktur pertrochanter $42 \%$

- Femurfraktur subtrochanter 1\%

Angaben zur Inzidenz differieren allerdings erheblich zwischen verschiedenen 
Ländern und Regionen von ca. 90 (Honam/Korea) bis über 650 Frakturen (Oslo/Norwegen) pro 100000 Einwohner mit einem Trendgefälle abnehmend von Nord nach Süd $[1,9,10,11,12,13,14]$.

Die Ursachen hierfür sind im Detail nicht geklärt. Amerikanische wie europäische Studien lassen bei über 65-jährigen eine Korrelation zwischen dem Breitengrad bzw. der Zahl der jährlichen Sonnenscheinstunden und der Häufigkeit von Schenkelhalsfrakturen annehmen [8]. Die vor allem in skandinavischen Ländern deutlich erhöhte Inzidenz wird unter anderem auf eine geschwächte Knochensubstanz infolge latentem VitaminD-Mangel durch verkürzte Sonnenscheindauer zurückgeführt. Dies kann analog in gleichem Maße auch für ältere Menschen in Pflegeeinrichtungen angenommen werden, die vor allem in den Wintermonaten nicht außer Haus kommen. Patienten mit hüftnahen Frakturen stammen überdurchschnittlich häufig aus betreuten Einrichtungen [5].

\section{Weitere Entwicklung}

Ingesamt lässt sich eine stetige Zunahme der Inzidenz in den letzten Jahrzehnten nachweisen. Zetterberg [16] eruierte z.B. für Schweden einen Anstieg der Frakturhäufigkeit zwischen 1965 und 1983 von $109 \%$ ! Dabei kann diese Steigerung nur zu etwa $20 \%$ durch die demografische Entwicklung mit ständiger Zunahme des Anteiles älterer Menschen in der Gesellschaft erklärt werden. Eine ähnliche Entwicklung ist auch für die Bundesrepublik zu erwarten. Im Jahre 2030 werden die 60-80-jährigen die stärkste Bevölkerungsgruppe bilden (Abb.2).

Bereits jetzt beträgt der Anteil der über 60-jährigen Patienten in unfallchirurgischen Kliniken ca. 50\%. Im zeitlichen Verlauf ist für diese Patientengruppe ein überproportionaler Anstieg im stationären Sektor zu beobachten mit fortschreitendem Trend.

\section{Ätiologie}

Anteilig betreffen nur ca. $2-3 \%$ der proximalen Femurfrakturen jüngere Patienten. Ursächlich erleiden diese Patienten in der Regel hochenergetische Traumen. Meist verursacht die Fraktur ein Sturz aus großer Höhe oder Kollisionen im Rahmen von Verkehrsunfällen. Durch eine axiale Krafteinleitung auf die gebeugte und abgespreizte Hüfte bricht dabei typi-

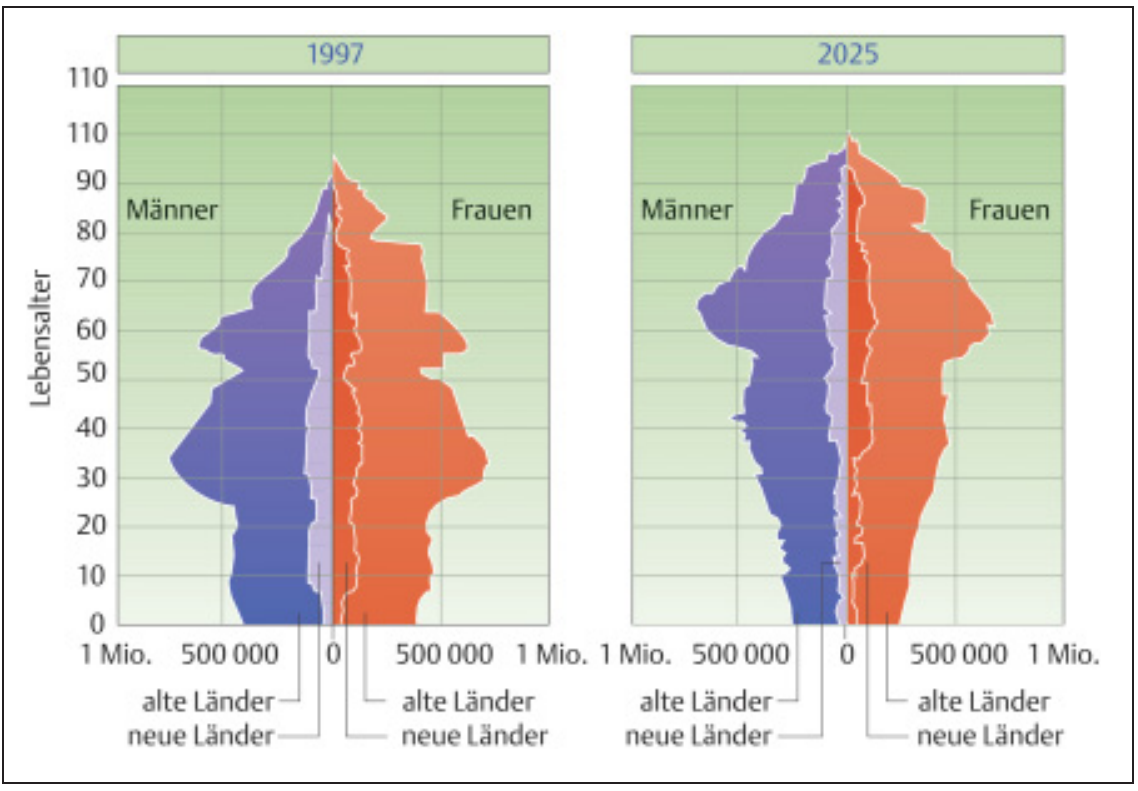

Abb. 2 Erwartete Bevölkerungsentwicklung in der BRD bis zum Jahr 2030 (Quelle: Ärztezeitung 2001; 1: 23).

scherweise der Schenkelhals mit Ausbildung einer dorsalen Trümmerzone [3].

Demgegenüber werden bei den deutlich häufigeren Frakturen im zunehmenden Lebensalter anamnestisch meist so genannte niedrigenergetische Mechanismen angegeben. Überwiegend sind es „banale Stürze“ im Alltagsleben aus dem Stand heraus. Infolge einer alterungsbedingt herabgesetzten mechanischen Belastbarkeit des Knochens führen der direkte Sturz auf den Trochanter major, eine plötzliche Abduktion in der Hüfte oder eine forcierte Außenrotation zur Fraktur. Die mechanische Resistenz des Skelettsystems ist dabei aber nicht alleine von der eigentlichen Knochenfestigkeit, sondern ebenso vom Zustand der Muskulatur und der neuromuskulären Koordination abhängig.

\section{Risikofaktoren, Risikoabschätzung}

Neben Art und Ausmaß des Unfallgeschehens lassen sich multiple weitere, teils vorgegebene, teils beeinflussbare Faktoren für die Frakturgenese eruieren [9].

\section{Lebensalter, Geschlecht}

Die proximale Femurfraktur ist, neben Wirbelkörperfraktur, proximaler Humerusfraktur und distaler Radiusfraktur, die typische Altersfraktur. 97-98\% werden jenseits des 50. Lebensjahres beobachtet (Abb. 3).
In einem Alter über 90 Jahren haben ca. $32 \%$ der Frauen und 17\% der Männer eine proximale Femurfraktur erlitten (11).

Nach Seibel [9] nimmt das relative Frakturrisiko mit jedem 5-Jahres-Lebensintervall um etwa Faktor 2 zu. Demnach hat jede heute 50-jährige Frau ein statistisches Risiko von etwa $15 \%$, im Laufe ihrer weiteren Lebenszeit eine proximale Femurfraktur zu erleiden. Die altersspezifische Inzidenz erreicht erwartungsgemäß ihr Maximum mit einer jährlichen Frakturhäufigkeit von $4 \%$ aller über 90 jährigen Frauen [18].

Ob der Faktor „Alter“ für sich alleine bereits Risikofaktor ist oder letztlich nur das Endprodukt weiterer alterskorrelierender Faktoren darstellt, ist nicht geklärt.

Auffallend ist das Überwiegen der Frauen im höheren Lebensalter. Das Geschlechtsverhältnis Männer/Frauen wird zwischen 1: 2,4 und 1: 4,2 für die BRD angegeben $[1,7]$. Die Häufung beim weiblichen Geschlecht wird zurückgeführt auf einen erniedrigten Anteil der Knochen- und Muskelmasse am Gesamtgewicht bei Frauen, klimakterisch bedingtem Hormonmangel und der höheren Lebenserwartung.

\section{Körpergewicht/ Körpergröße}

Niedriges Körpergewicht bzw. niedriger Bodymass-Index (BMI) sind verbunden mit einer höheren Frakturgefährdung. 
Neben eigentlicher Fehl- oder Mangelernährung mit erhöhtem Osteoporoserisiko haben wahrscheinlich mechanische Faktoren, wie das Ausmaß des Fettpolsters oder die Dicke des Muskelmantels in der Rolle als Stoßdämpfer eine Bedeutung [9].

\section{Osteoporose}

Osteoporose bezeichnet den Verlust an Knochensubstanz, bedingt durch ein Missverhältnis aus vermehrtem Knochenabbau oder vermindertem Knochenneuanbau. Sie führt im Erkrankungsverlauf zu einer zunehmenden mechanischen Schwächung des Knochens mit erhöhter Frakturgefährdung. Ca. 6 Millionen Menschen sind alleine in Deutschland betroffen. Durch Zunahme des Anteiles älterer Menschen ist in den nächsten Jahrzehnten mit einem weiteren Anstieg zu rechnen.

Zwischenzeitlich sind viele Ursachen bekannt, die die Entstehung einer Osteoporose begünstigen. Neben vorgegebenen, anlagebedingten Faktoren, wie familiäre Belastung, weiße Rasse, Körperbau und bei Frauen Zeitpunkt von Menarche und Menopause, finden sich zahlreiche weitere, auch therapeutisch beeinflussbare Risiken.

Dazu zählen zahlreiche Erkrankungen ebenso wie Risiken, entstehend aus der individuellen Lebensführung (Tab.1).

\section{Sturz und Sturzneigung}

Nach der Osteoporose ist in höherem Lebensalter vor allem die erheblich gesteigerte Sturzneigung in der Frakturengenese von großer Bedeutung. Vor allem die Geriatrie befasste sich in den vergangenen Jahren eingehend mit der Erforschung von Ursachen und Folgen von Stürzen.

\section{Dazu einige Fakten $[17,19,20]$ :}

- Ca. ein Drittel aller über 65-jährigen Patienten haben in den letzten $90 \mathrm{Ta}-$ gen vor dem Frakturereignis mindestens einen weiteren Sturz erlitten.

- Von Bewohnern aus Alten- und Pflegeheimen, sowie von allen über 80-Jährigen stürzt jährlich mindestens jeder Zweite.

- $10 \%$ der Stürze verursachen behandlungsbedürftige Verletzungen, davon über $50 \%$ Frakturen

Ca. 95\% der Stürze ereignen sich in der Wohnung, davon $80 \%$ alleine in Bad,
Tab. 1 Die wichtigsten Risikofaktoren für die Entwicklung einer primären oder sekundären Osteoporose (n.9)

Familiäre Belastung:

- Osteoporose bei Eltern/Geschwistern

Umwelt und Lebensführung:

- Bewegungsmangel

- Fehlernährung mit Kalziummangel

- geringe Sonnenexposition

- Rauchen

Alkoholabusus

Sexualhormone:

- weniger als 30 Jahre zwischen Menarche und Menopause

- Menopause vor 45. Lebensjahr

— längere Östrogenmangelzustände

- Hyperprolaktinämie

- Hypogonadismus

iatrogene Faktoren:

- Glukokortikoid-Therapie > 6 Mon.

- Heparin-Langzeitgabe

- Antiepileptika

Erkrankungen:

n primärer Hyperparathyreoidismus

- unbehandelte Hyperthyreose

- Hyper- und Hypocortisolimus

a Plasmozytom und andere maligne Tumoren (Tumorosteopathie)

- Rheumatoide Arthritis

- Malabsorption (z.B. Crohn, Sprue, post Gastrektomie-Syndrom, u. a.)

Toilette und Flur. Weniger als 5\% der Stürze erfolgen außer Haus.

Dabei sind es vor allem innere Ursachen im Zusammenhang mit dem beeinträchtigtem Gesundheitszustand, die bereits bei geringfügig äußeren Anlässen zur Fraktur führen (Tab.2).

Sturzauslösend wirken im Alter zum Einen gehäuft auftretende internistische Erkrankungen ebenso wie Bewegungseinschränkungen bei neurologischen oder orthopädischen Krankheitsbildern. Zum Anderen verursachen die herabgesetzte Reaktionszeit, verminderte Muskelmasse und -kraft sowie strukturell degenerative Veränderungen am Gehirn Störungen der neuromuskulären Koordination mit Beeinträchtigung des Gangablaufes. In der Gerontologie ist deshalb die Qualität der Gang-Motorik ein zentraler Maßstab für den allgemeinen Gesundheits- und Leistungszustand eines älteren Menschen.

Bereits einfache Screening-Verfahren lassen dabei den gefährdeten Personenkreis im Vorfeld erkennen, um möglichst dem Frakturereignis im Sinne der Präventivmedizin zuvorzukommen (Tab.3).
Tab. 2 Übersicht zu Sturzrisiken

Gangstörung/gestörte Koordination

- Parkinson, Apoplex, Muskelschwäche, Polyneuropathie

Balancestörung

- Schwindel, Orthosthase, Herz-Rhythmusstörungen, Bluthochdruck

muskuläre Kraft und Leistungsminderung der Beine

funktionelle Einschränkungen an der unteren Extremität

- Arthrose, Kontrakturen, Beinlängendifferenz, Fehlstellung

Medikation mit mehr als 4 Pharmaka

- insb. Neuroleptika, Antidepressiva, Benzodiazepine, Antikonvulsiva

geistige Leistungsminderung

- Demenz/psychomotorische Unruhe

zunehmende Gehstreckenreduktion

Sehschwäche

ADL Defizit, Gebrauch von Gehhilfen

- Hinweis auf präexistente Einschränkungen

positive Sturzanamnese

a zwei oder mehr im Jahr

psychische Faktoren

Angst vor einem Sturz, Depression, Alkohol-Krankheit

hohes Alter ( $>85$ Jahre)

\section{Prophylaxe}

Die proximale Femurfraktur als Sturzfolge im Alter ist mehr als ein chirurgisch zu therapierendes Ereignis. Der Sturz ist Indikator für den drohenden Autonomieverlust des alten Menschen. Er kennzeichnet ein akutes Problem ( Schwindel, Orthostase), das Fortschreiten einer chronischen Erkrankung (Parkinson, Demenz) und die Verschlimmerung von Altersveränderungen (Visusverlust, Muskelatrophie) $[20,21]$.

Für viele ältere Patienten bedeutet eine hüftnahe Femurfraktur eine einschneidende Gefährdung und oft eine Wende in ihrer bisherigen Lebensführung.

\section{Hierzu einige Schlaglichter:}

- Nach überstandenen Frühkomplikationen mit erhöhter Sterblichkeit von 10,4\% noch während der Behandlung in Akut- und Reha-Klinik [5] besteht auch über diesen Zeitraum hinweg eine hohe Gefährdung. Binnen eines Jahres sind 29-38\% der Patienten (intrakapsuläre vs. extrakapsuläre Frakturen) verstorben [26].

- Nach 1 Jahr sind nur ca. 63\% der Patienten wieder in ihrer alten Wohnum- 
Tab.3 Einfacher Risiko-Score zur Erkennung der Sturzgefährdung. Bei zwei oder mehr positiven Hinweisen sollte präventiv eine ärztliche Intervention erfolgen (22)

Wie erkenne ich Gehstörungen mit Sturzgefahr?

Hinweise auf Sturzgefahr nach dem PISA-Projekt der Aerpah-Kliniken Esslingen-Kennenburg und Ilshofen (nach M. Runge: Gehstörungen, Stürze, Hüftfrakturen. Steinkopff-Verlag Darmstadt 1998; www.mobility-clinic.de)

\begin{tabular}{|c|c|c|}
\hline Was fällt auf? & & Erklärung \\
\hline $\begin{array}{l}\text { Gangbild ist } \\
\text { - sehr langsam oder } \\
\text { - sehr unregelmäßig }\end{array}$ & O & $\begin{array}{l}\text { Auffällig sind kleine, unregelmäßige Schritte, vielleicht sogar mit häufigem Stolpern/Straucheln. } \\
\text { Hangeln/Greifen nach jedem Halt ist ebenfalls ein Zeichen für Sturzgefahr. }\end{array}$ \\
\hline Balancestörungen & $\bigcirc$ & $\begin{array}{l}\text { Wenn man nicht in der Lage ist, } 10 \text { Sek. in folgender Weise zu stehen: Füße stehen in einer Linie } \\
\text { hintereinander, Hacke des vorderen berührt spitze des hinteren Fußes (= Tandemstand). }\end{array}$ \\
\hline Kraft- und Leistungsminderung Beine & $\bigcirc$ & $\begin{array}{l}\text { Sturzgefährdet ist jeder, der nicht schneller als in } 12 \text { Sekunden } 5 \times \text { aus einem Stuhl üblicher Höhe } \\
\text { aufstehen kann, ohne sich dabei mit den Armen abzustützen. }\end{array}$ \\
\hline $\begin{array}{l}>4 \text { verschiedene Medikamente pro } \\
\text { Tag oder bestimmte Medikamente }\end{array}$ & $\bigcirc$ & $\begin{array}{l}\text { Wer mehr als vier Medikamente pro Tag braucht, ist sturzgefährdet. Manche Medikamente können } \\
\text { nicht abgesetzt werden, obwohl sie die Sturzgefahr erhöhen. }\end{array}$ \\
\hline zwei oder mehr Stürze im letzten Jahr & O & Jeder Sturz sollte ärztlich abgeklärt werden, auch wenn keine Verletzungen entstanden sind. \\
\hline geistige Leistungsminderung & O & Demenzkranke und verwirrte Patienten sind besonders gefährdet, wenn sie viel umherlaufen. \\
\hline Sehen verschlechtert & $\bigcirc$ & Besonders gefährlich, wenn man mit einem Auge deutlich schlechter sieht als mit dem anderen. \\
\hline Probleme an Beinen und Füßen & $\bigcirc$ & $\begin{array}{l}\text { z. B. Schmerzen an Hüfte, Knie oder Fuß, wobei plötzlich einschießende Schmerzen besonders } \\
\text { gefährlich sind. }\end{array}$ \\
\hline Gehhilfe erforderlich & O & Gang ist mit Gehstock, Gehwagen etc. subjektiv oder objektiv sicherer. \\
\hline $\begin{array}{l}\text { Beweglichkeit und Gehleistung wer- } \\
\text { den allmählich immer schlechter }\end{array}$ & O & $\begin{array}{l}\text { Wenn jemand dazu neigt, seinen Bewegungsradius immer mehr einzuschränken, ist dies oft ein } \\
\text { Hinweis auf eine Gehstörung. }\end{array}$ \\
\hline Osteoporose & ○ & $\begin{array}{l}\text { Bei verminderter Knochenfestigkeit kommt es schneller zu Knochenbrüchen (allerdings gibt es } \\
\text { auch ohne Osteoporose Brüche von Hand und Oberschenkel). }\end{array}$ \\
\hline
\end{tabular}

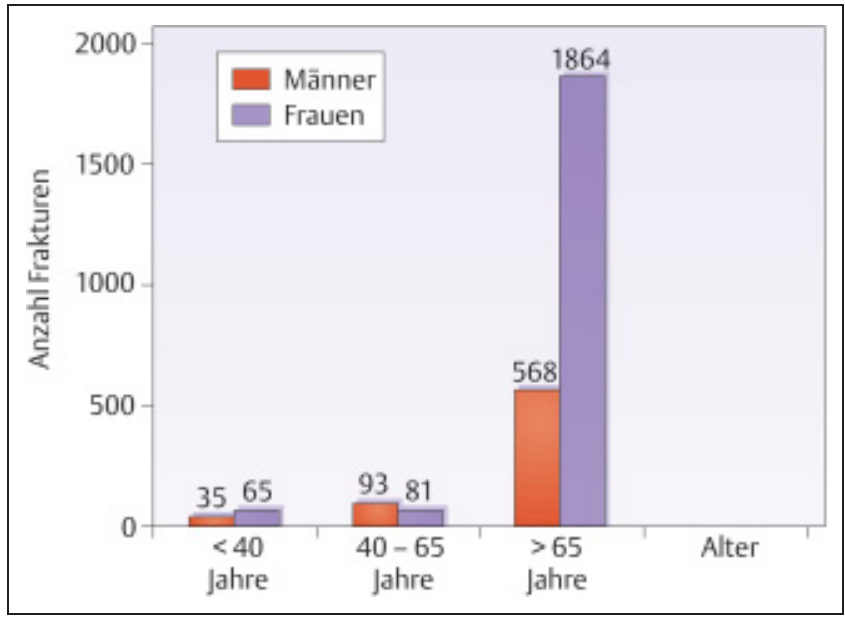

Abb. 3 Altersverteilung der Schenkelhalsfrakturen in Deutschland im Jahr 1996 [7].

gebung, ca. $18 \%$ benötigen eine erhöhte pflegerische Betreuung [5]. Ein Drittel aller Patienten bleibt dauerhaft pflegebedürftig [7].

- Bedingt durch die demographische Entwicklung wird ein Fallanstieg bis 2030 um das Dreifache erwartet [24].

- Im Durchschnitt 3,3 Jahre später haben sich $62 \%$ der Patienten mit Schenkelhalsfraktur, $72 \%$ mit pertrochanterer Fraktur eine meist identische Zweitfraktur auf der Gegenseite zugezogen [23].
Analog der multifaktoriellen Genese proximaler Femurfakturen ergeben sich mehrere Ansätze prophylaktisch einzugreifen:

\section{Medikamentös \\ - Physikalische Therapie \\ - Stoßabsorption}

An erster Stelle gilt es natürlich akute internistisch und neurologische Erkrankungen möglichst optimal und ursächlich zu therapieren.

Evidenzbasierte Studien belegen den Sinn der medikamentösen Intervention bei bestehenden Osteoporoserisiken [in 7]. Alleinige Kalzium- und Vitamin D-Verabreichung an Altenheimbewohnern senkte die Frakturinzidenz um 43\%, die dreijährige Gabe von neueren Biphosphonaten an postmenopausale Frauen um $40 \%$.

Zahlreiche prospektive Studien zeigen den prophylaktischen Wert jeglicher Bewegungsaktivitäten mit Minderung des Sturzrisikos bis knapp 50\% [in 21], ausgehend von Tanzen bis hin zu Tai Chi und spezifischem Gleichgewichts- oder Krafttraining der unteren Extremität. 


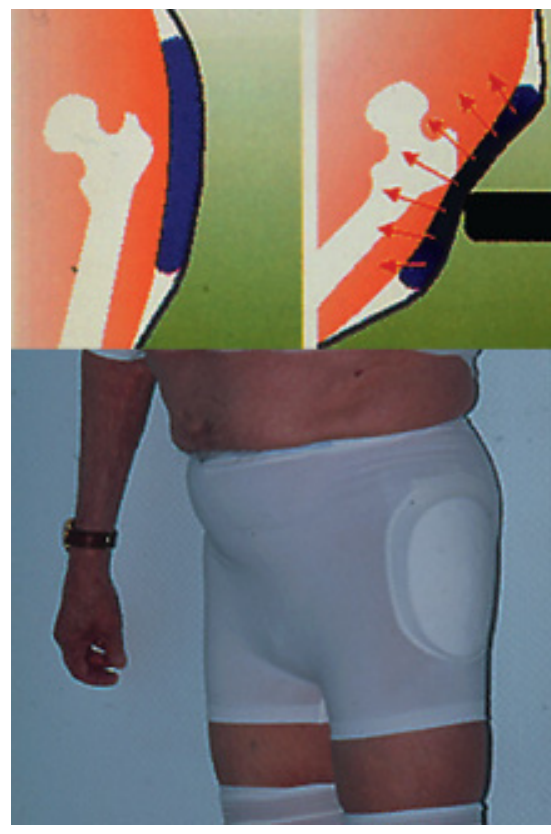

Abb. 4 Patient mit anliegendem Hüftprotektor $\left(\right.$ Savehip $\left.^{\circledR}\right)$. Schematische Darstellung der Wirkungsweise. Stoßabsorption durch rutschfest eingearbeitetes Hartschalenpolster.

Bei einem Sturz treten bis 5-mal so hohe Energien auf als nötig sind, ein normales Femur zu brechen. Für die Frakturentstehung scheint deshalb vor allem das Ausmaß der Absorption der Sturzenergie durch umgebende Weichteile von Bedeutung zu sein. Spezielle Hüftprotektoren (Abb.4) sind in der Lage, diese Stoßabsorption zu gewährleisten und können das Frakturrisiko über $50 \%$ reduzieren $[6,7]$. Zielgruppe sind vor allem hochgefährdete Patienten, z. B. in betreuten Einrichtungen, um die Restmobilität zu erhalten. Die schweizerische Bundesanstalt für Unfallverhütung kam in einer Kosteneffektivitätsanalyse bei Einsatz von Hüftprotektoren in Altenheimen auf jährlich ca. 35 Mio. SFr. Einsparpotential.

In der BRD wäre hierdurch eine jährliche Fallzahlreduktion von ca. 10000 proximalen Femurfrakturen möglich [2].
$\mathrm{Zu}$ erwähnen wären noch entsprechend „altengerechte“ Zurichtungen der Wohnumgebung mit Beseitigung von Stolperfallen und Hindernissen, Anbringen von zusätzlichen Haltegriffen und möglichst guter Beleuchtung.

\section{Literatur}

${ }^{1}$ Pauschert R, Niethard F, Schöning B, Lurz G. Letalität nach Frakturendes coxalen Femurendes im fortgeschrittenen Alter. Dt. Ärzteblatt 1996; 93/3: $102-107$

${ }^{2}$ Meyer G, Warnke A, Kapfer E, Becker C. Pr̂tà-porter Hüftprotektoren beugen vor. Geriatrie Journal 2000/9

${ }^{3}$ Kutschka-Lissberg F, Schildhauer TA, Kollig E, Muhr G. Die Osteosynthese der medialen Schenkelhalsfraktur. Chirurg; 2001; 72: $1253-1265$

${ }^{4}$ Cree AK, Nade S, Aust. How to predict return to community after fractured proximal femur in the elderly. NZJ Surg.1999; 69(10): $723-725$

${ }^{5}$ Schurch MA, Rizzoli R, Mermillod B, Vasey H, Michel JP, Bonjour J. A prospective study on sozioeconomic aspects of fracture of the proximal femur. J Bone Miner Res 1996; 11(12): 1935-1942

${ }^{6}$ Lotz JC, Hayes WC. The use of quantitative computed tomography to estimate risk of fracture of the hip from falls. J Bone Joint Surg Am 1990; 72(5): 689-700

${ }^{7}$ Pfeifer M, Wittenberg R, Würtz R, Minne HW. Schenkelhalsfrakturen in Deutschland. Dt. Ärzteblatt 2001; 98: 1751 - 1757

${ }^{8}$ Jacobsen SJ, Goldberg J, Miles TP, Brody JA, Stiers W, Rimm. Regional variations in the Incidence of Hip fractures: US white women aged 65 years and older. J Am Med Ass 1990; 264: 500-502

${ }^{9}$ Seibel MJ. Evaluation des osteoporotischen Frakturriskos; Dt. Ärzteblatt 2001; 98/25: $1681-1689$

${ }^{10}$ Nydegger V, Rizzoli R, Rapin CH, Vasey $\mathrm{H}$, Bonjour JP. Epidemiology of fractures of the proximal femur in Geneva: Incidence, clinicaland social aspects; Osteoporos Int 1991; 2(1): $42-47$

${ }^{11}$ Gallagher JC, Melton LJ, Riggs BL, Bergstrath E. Epidemiology of the fractures of the proximal femur in Rochester, Minnesota; ClinOrtho 1980; 150: 163-171

12 van Staa TP, Dennisio EM, Leufkens HG, Cooper C. Epidemiology of fractures in England and Wales; Bone 2001; 29(6): 517-522

${ }^{13}$ Baron JA, Karagas M, Barrett J, Kniffin W, Malenka D, Mayor M, Keller RB. Epidemiolgy 1996; 7(6): 612-618

${ }^{14}$ Sander GC, Seeman E, Ugoni AM, Pasco JA, Matin TJ, Skoric B, Nicholson GC, Kotowicz MA. Age- and gender-spezific rate of factures in Australia: a population based study; Osteoporos Int 1999; 10(3): 240-247
${ }^{15}$ Verettas DA, Galanis B, Kazakos K, Hatziyiannakis A, Kotsois E. Fractures of the proximal part of the femur in patients under 50 years of age. Injury 2002; 33(1): $41-45$

${ }^{16}$ Zetterberg C, Elmerson S, Anderson GB. Epidemiology of hip fractures in Goteborg, Sweden, 1940-1983; Clin Orthop 1984; 191: $43-52$

${ }^{17}$ Fleischer SE. Der „Mobility outcome test“, Prädiktoren für Mobilität nach proximaler Femurfraktur im Alter. Dissertation Uni Ulm 2000

${ }^{18}$ Martin AD, Silverthorn KG, Houston CS, Berhardson S, Wajda A, Roos LL. The incidence of fracture of the proximal femur in two million Canadians from 1972 to 1984. Projektions for Canada in the year 2006.

${ }^{19}$ Falck I. Die Bedeutung von Stürzen in der Geriatrie; Zeitschrift f Gerontologie 1983: 16(6): $254-259$

${ }^{20}$ Becker C, Scheible S. Stürze und sturzbedingte Verletzungen älterer Menschen; Fortschritte in der Medizin 1998; 166(32): $22-29$

${ }^{21}$ Bundesarbeitsgemeinschaft Senioren, BAGSO Nachrichten online 03/2001; http: www.bagso.de

${ }^{22}$ Runge M. Gehstörungen, Stürze, Hüftfrakturen; 1998; Steinkopf-Verlag Darmstadt

${ }^{23}$ Schroeder HM, Petersen KK, Erlandsen M. Occurene and incidence of the second hip fracture; Clin Orthop 1993; 289: 166-169

${ }^{24}$ Kannus P, Niemi S, Parkkari J, Palvanen M, Vuori I, Jarvinen M. Hip fractures in Finnland between 1970 and 1997 and prediction for the future; Lancet 1999; 353(9155): $802-$ 805

${ }^{25}$ Jones G, Nyugen T, Sambrook PN, Kelly PJ, Gilbert C, Eisman JA. Symptomatic fracture incidence in elderly men and women: the Dubbo Oseoporosis Epidemiology Study (DOES); Osteoporosis Int; 1994; 4(5): $277-282$

${ }^{26}$ Keene GS, Parker MJ, Pryor GA. Mortality and morbidity after hip fracture; BMJ 1993; 13 : 307(6914); $1248-1250$

\section{Dr. med. Rolf Balk}

Ltd. Oberarzt

Prof. Dr. med. Friedrich Hahn

Ärztl. Direktor

Dr. med. Bogdan Tarcea

Assistenzarzt

Ostalb-Klinikum Aalen

Chir. Klinik II: Unfall- Hand- und

Wiederherstellungschirurgie

Im Kälblesrain

D-73431 Aalen 